\title{
CiteSpace Based Dynamic Visualization Analysis of Domestic Researches on Cross - Cultural Communication 1996-2016
}

\author{
Xiao-ya GAN ${ }^{1, a,{ }^{*}}$ and Ying-Xue HUANG $^{2, b}$ \\ ${ }^{1,2}$ Foreign Languages School of Wuhan University of Science and Technology, Wuhan \\ 430065, China \\ aganxiaoya2004@126.com, ${ }^{b} 1291606981 @ q q . c o m$
}

Keywords: CiteSpace, Cross-Cultural Communication, Hot Issues.

\begin{abstract}
The paper has collected data of cross-cultural communication related research papers published on 12 domestic authoritative journals since 1996 and has conducted analysis based on knowledge mapping. By means of CiteSpace, the widely used software in knowledge mapping, the paper has detected hot issues in the research of cross-cultural communication in China, tracked the evolution of hot issues and identified the most fruitful authors in the field. The study displays that the three hottest issues are the cross-cultural communicative competence, foreign language teaching and cultural differences. Furthermore, the empirical research has begun to occupy the mainstream trend currently.
\end{abstract}

\section{Introduction}

As a newly-introduced subject, cross-cultural communication isn't developed long in China until some domestic scholars gradually begin to pay much attention to it at the beginning of the $80 \mathrm{~s}$ of last century. In recent years, cross-cultural communication symposium has increased and the journal Cross-Cultural Communication Research has been set up; meanwhile, the academic dialogues between domestic and abroad have become increasingly frequent, and the amount of research papers on cross-cultural communication has been improved noticeably. However, as Chen Guoming (2009:241) has pointed out, literature on cross-cultural communication studies are still chaotic and lack of a holistic view. Hence, this study will take advantage of CiteSpace, the effective software in knowledge mapping, systematically carding and analyzing the China studies of cross-cultural communication in the past 20 years to clear the dynamic direction of hot issues in this field and eventually to promote the development of cross-cultural communication research in China.

\section{Sources of Data}

To increase the objectivity and persuasiveness, the paper has taken advantage of CiteSpace II, the visualization software developed by Dr. Chen Chaomei from Drexel University, USA. The study collects article data from the 12 kinds of core journals in domestic foreign language research. Only periodical papers are included, while some meetings reports, notices, dynamics and other non-academic papers are excluded by the author in data processing. The 12 kinds of foreign language core journals are listed in Figure 1. The author retrieve literature data of cross-cultural communication research papers published on the above listed 12 kinds of journals as data sources. Specifically, set "advanced search" in retrieval condition column, enter "Cross-cultural Communication" in "theme" column, enter "January 1, 1996 to May 8, 2016" in time 
limit, select the above listed 12 journals in the source of journal, and then 315 pieces of effective literature are obtained. The data of the last update time is May 8, 2016. The statistical data are displayed in figure 1:

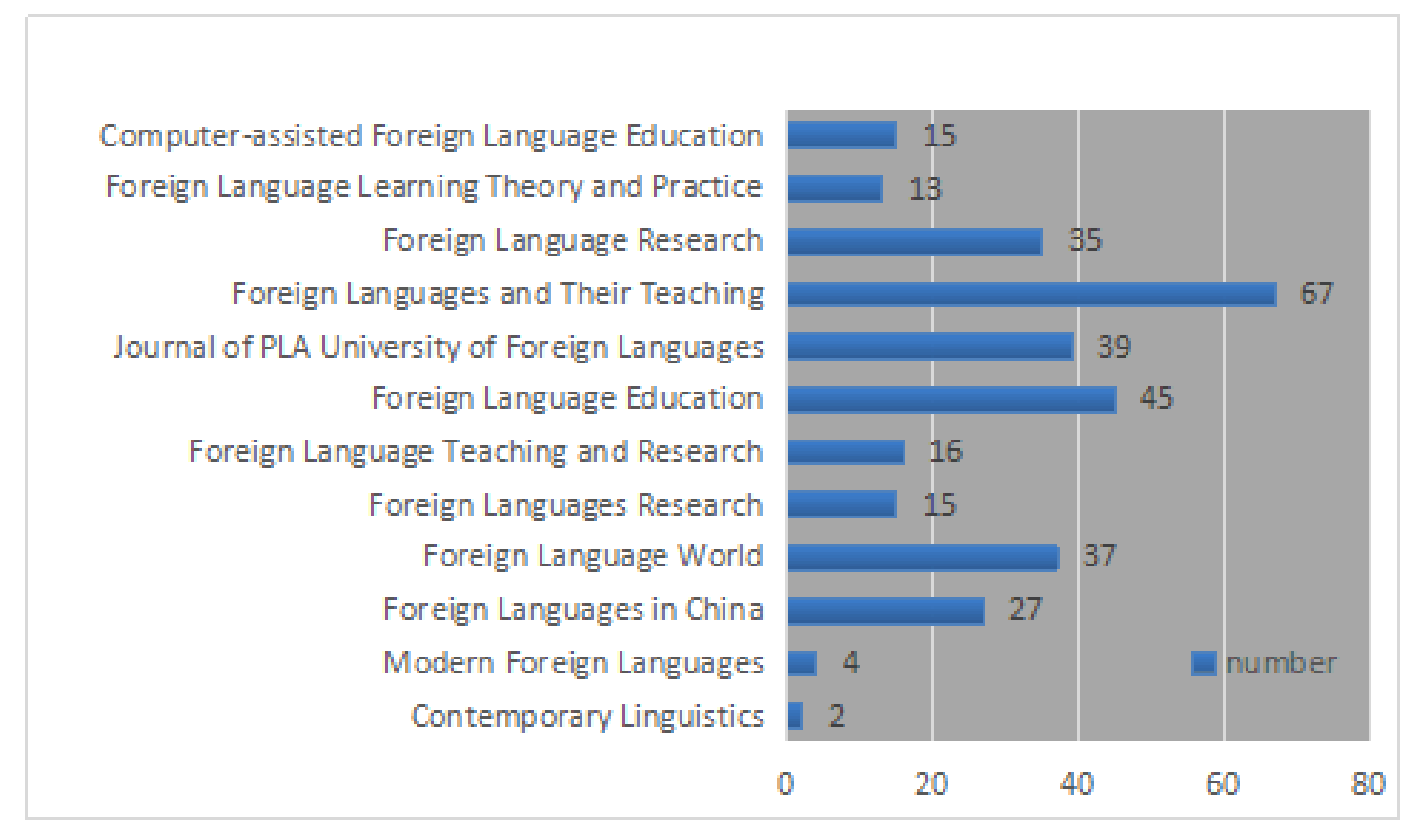

Figure 1. Statistical information of papers on cross-cultural communication published on 12 core journals 1996-2016

As shown in Figure 1, there are 315 research papers published on the above listed core journals from 1996 to 2016, in which Foreign Languages and Their Teaching are far ahead of other journals, including 67 articles published; then Foreign Language Education, Foreign Language Research, Foreign Languages in China, Journal of PLA University of Foreign Languages and Foreign Language World have covered more than 35 articles respectively. It is evident that foreign language teaching has paid more concern of cross-cultural communication in the field of foreign language research.

\section{Data Analysis and Discussion}

The author uses the cluster analysis method to draw the hot issues knowledge mapping of cross-cultural communication research in China. Specifically, enter "1996 to 2016" in "Time" column, with one year as a time slice, select "Title", "Abstract", "Author Keywords" and "Keywords plus" in "Term Source", select "Keywords" in the "Node Type" and keep the parameters unchanged. Based on these requirements, the software automatically extracts 24 clusters. The clusters with frequencies equal to or larger than 4, which are clusters $0-13$ are discussed in the paper, while the rest are omitted due to the comparatively low frequency and slight reference value. As shown in Figure 2, the cluster value Q Modularity is 0.8943 , the cluster internal similarity index, namely, Silhouette value is 0.5904 , which indicates that module clustering is obvious and the homogeneity within the cluster is evident since the clustering information is considered to be valid if Silhouette value is more than 0.5 . 


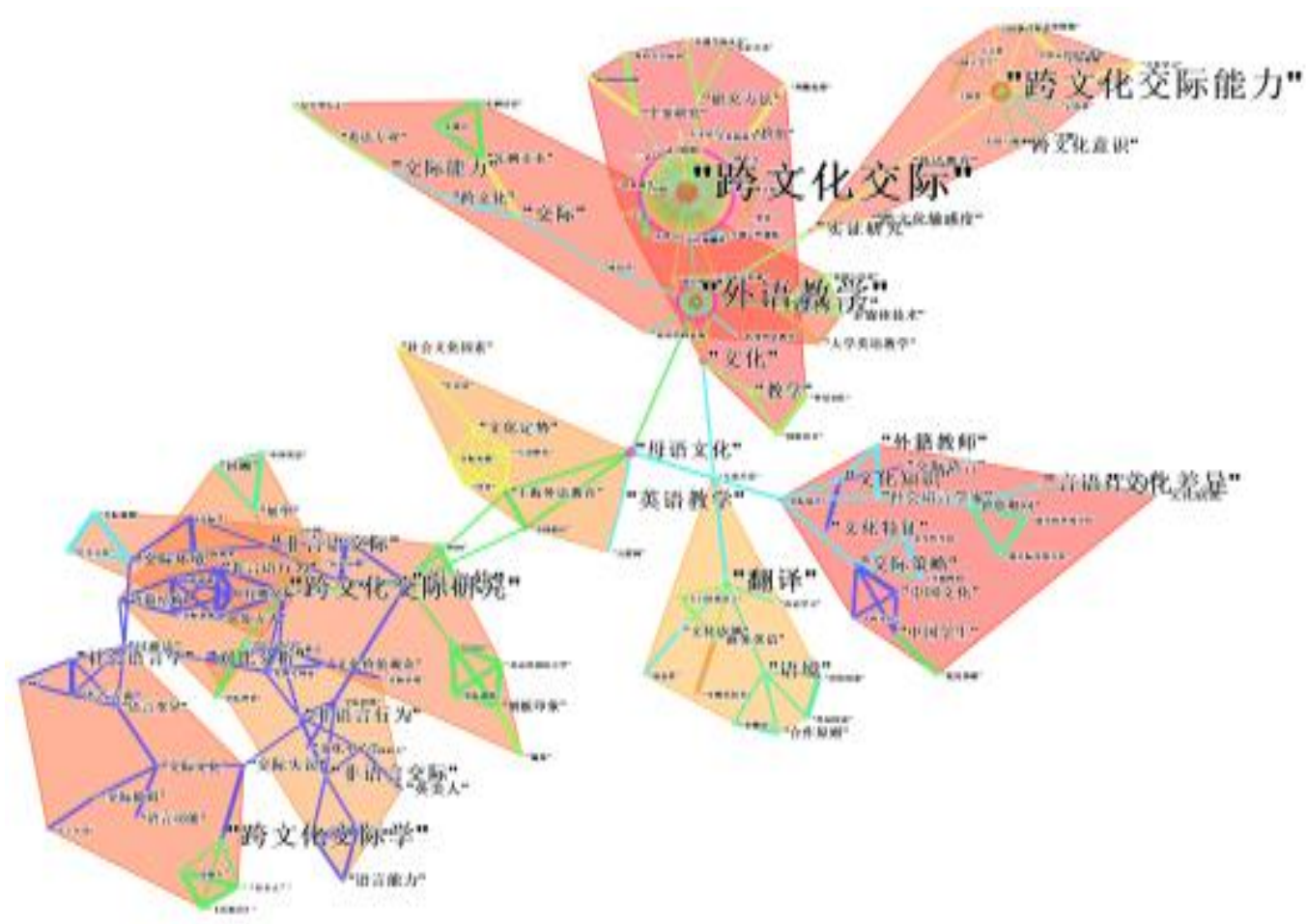

Figure 2. Journal articles in CNKI (1996-2016): the key words co-occurrence knowledge mapping

Figure 2 is the effect diagram of clusters and the detailed frequency statistics of the key words included in each cluster can be seen in a table. Because of limited space, the table is not listed here. In this paper, only the first 3 clusters are discussed in detail, namely, cluster 0 (cross-cultural communication), cluster 1(foreign language teaching), cluster 5(cultural differences) and then they will be elaborately analyzed respectively in the following parts.

\section{Cross-cultural Communication Research}

Cross-cultural communication ("kua wen hua jiao ji xue" in Chinese), as an independent discipline, has originated from the term "cross-cultural/intercultural communications", which really began to appear in English world about half a century ago (Chen Guoming, 2010:141-148). Edward T Hall firstly put forward the term "cross-cultural/intercultural communication" in "silent language" published in 1959.

The primary study of cross-cultural communication is the study of the distinction between cross-cultural communicative competence and cross-cultural competence. As Hu Wenzhong (2013:3) states that, Cross-cultural communicative competence itself is very complicated, and the international academic has discussed on this issue for more than half a century. According to the literature collected, different people hold different opinions on this point, but the most wildly accepted three can be presented as following: cross-cultural communicative competence is equivalent to cross-cultural competence, cross-cultural communicative competence represent more than cross-cultural competence and cross-cultural competence represent more than cross-cultural communicative competence.

Specifically speaking, the representative figure who holds the opinion of "equivalence" is Chen Guoming. He believes that these two are the same concept, and cross-cultural communicative competence is cross-cultural competence; they are the same and one can be totally replaced by another one. Yang Ying and Zhuang Enping 
also think that "regarding cross-cultural communicative competence as cross-cultural competence are conducive to the liberation of our ideas from the narrow perspective of language communication, so that in the development process of cross-cultural communication competence, people will not only pay much attention to the ability of language communication, but also the importance of the cross-cultural awareness, the ability of thinking, non-verbal communication and communication strategies and so on." (2007:16); the representative figure holding the opinion that cross-cultural communicative competence represent more than cross-cultural competence is Wen Qiufang, whose main theory is cross-cultural communicative competence contains cross-cultural competence. She believes that communicative competence and cross-cultural competence form cross-cultural communicative competence. According to Wen (2004:175), communicative competence includes linguistic competence, pragmatic competence and versatility; cross-cultural competence includes the sensitivity to cultural differences, the tolerance of cultural differences and the flexibility to deal with cultural differences. The last viewpoint is that cross-cultural competence includes cross-cultural communicative competence; however, the former two hold the dominant position in the field of foreign language linguistics.

Secondly, it is the study of the definition of cross-cultural communicative competence. Hu Wenzhong (2013:4) states that many scholars have defined the terms of cross-cultural communication competence; although there is no definition universally accepted, scholars have generally acknowledged that 'cross-cultural communicative competence refers to the ability to communicate effectively with people with different cultural backgrounds'. So it can be considered that scholars have reached a certain consensus on the definition of cross-cultural communication competence and its main components.

\section{Cross-Cultural Communication and Foreign Language Teaching}

Presently the foreign language community has paid great attention to cross-cultural communicative competence. As a key component of cross-cultural communicative competence, the concept "cross-cultural sensitivity" is firstly proposed by Bennett in 1984. However, the domestic research on cross-cultural sensitivity started late (Joe Huanrun, 2015:15). In China, theoretical researches on cross-cultural sensitivity are rare, which mainly concentrate on the cross-cultural sensitivity analysis, the relationship between the five levels of cross-cultural sensitivity and the use of cross-cultural/intercultural sensitivity test scale (Intercultural Sensitivity Scale, referred to as ISS). ISS, with a high reliability, which is developed by Chen and Starosta (2000), is mostly used in empirical researches.

In recent years, foreign language teachers have put forward a variety of different culture teaching modes. Xu Lisheng, Sun Shunv (2014) used the computer-dominated strategy to train students' communicative competence in College English Teaching. They put forward "classroom teaching mode, individual learning mode, cooperative learning mode, virtual training mode and Internet collaborative teaching mode and some other different teaching modes that can be used in the process of knowledge acquisition, motivation training, skills training and cross-cultural communication practice" to improve the students' cross-cultural competence. Pan Bingxin, Rong Jun (2000:30-33) used email-assisting foreign language teaching methods to conduct an empirical study on 63 students from both China and America. Liu Meihua, Liu Shisheng (2015) made an interview study of two junior students and the results showed that subjects thought it was difficult to integrate into the local cultural exchange. 


\section{Cultural Differences}

Cultural differences, refers to people who are from different cultural background and speak different language perform differently in their traditional customs, values, religious beliefs, thinking and other aspects. Culture is a word that is difficult to explain. In the book called crossing cultural barriers, Hu Wenzhong (2002:34-35) described culture as something that contains the history and also reflects the reality; contains material object and the system and concept as well; owns the stability of the objective existence and also the initiative of it; covers characteristics of a group and also represents regional differences and individual differences. Based on the perspective of cross-cultural communication, Zhang Zhanyi (1984) firstly proposed that the culture is divided into knowledge culture and communication culture because of the role of cultural factors in communication. He (1990:22) stated that knowledge culture refers to those language and non-language cultural factors which do not directly affect accurate transformation of information to each other (that is, causing deviation and misunderstanding) when two people with two different cultural backgrounds communicate; whereas communicative culture refers to those which would have a direct impact on information transformation. The concept of "knowledge culture" and "communicative culture" has aroused great attention in the field of foreign language teaching and it gradually has been widely accepted by scholars.

Owing to cultural differences, there are three main problems in cross-cultural communication activities: the ethnocentrism, cultural stereotypes and the paradox of cross-cultural communication. Cultural stereotypes, as the most widely used way to deal with cultural differences, is the most basic and also the most critical issues affecting cross-cultural communication. Stereotype refers to members of one group having a simple view to members of another one (and thus it initially owned "derogatory" features). Its premise is that members of the same group have similar characteristics (Zuo Xiaoyuan, 2000:102). Stereotypes(i.e., cultural stereotypes) in cross-cultural communication refers that people have a simple view to the nationals or the countries with different cultural background. In the public opinion, Walter Lippmann firstly pointed out that it is impossible for people to know everything in a complex living environment and the social environment where they live. In order to save time, they will fit someone or something with the same characteristics into a pre-specified prototype. On the one hand, cultural stereotypes reduce the burden of cognition, which helps people to obtain the most heterogeneous cultural information in the shortest time with the minimum effort; On the other hand, it is very easy to produce "racism" if the negative aspects of the heterogeneous culture or the positive aspects of the mother tongue culture are overdramatized, which would impede cross-cultural communication, and even lead to ethnic conflicts. There are both advantages and disadvantages for cultural stereotypes. Gao Yihong called this contradiction "the paradox of cross-cultural communication". The research of cultural stereotypes in the foreign language world mostly focus on the advantages and disadvantages of cross-cultural communication. Wang Lu (2008) analyzed the function of cultural stereotypes in cross-cultural communication and explored how to transcend cultural stereotypes to achieve successful cross-cultural communication. Similarly, Lin Biling (2011) proposed that we were supposed to treat cultural stereotypes with a dialectical attitude. Jia Lei (2015) put forward that effective cross-cultural communication can be achieved by establishing a certain cultural stereotypes to generalize the cultural differences and eliminate communication barriers caused by simple, one-sided 
stereotypes. In this way, cultural stereotypes are always constantly updated. It is known that scholars have had an objective attitude to cultural stereotype and future studies would probably focus on it.

In addition, some cultural phenomenon caused by cultural differences such as the vacancy phenomenon has also become the concern of the majority of scholars. According to the Russian vacancy theory school, people always meet something that they cannot understand or feel confused when the text they read are from different cultures, which is the characteristic of the this national cultural elements embodied in the text and called the vacancy phenomenon (Liu Hong, 2005). The research of our country in this area is mainly related to the study of lexical vacancy, vacancy phenomenon and translation studies. Another cultural phenomenon is known as "precedent phenomenon", which is mainly presented as precedent situation, precedent text, precedent name and precedent discourse and aims to promote a communicator to cognize foreign culture. It is often regarded as the core part of a nation's cognitive database. In this aspect, the research in our country is mainly concentrated in the Russian language research, such as the study on the precedent phenomenon and cross-cultural communication in Russian advertising texts (Gu Qian, 2011).

Besides, some scholars such as Gao Yongchen, Liu Hong, Hu Chao also overcome the communication barriers caused by cultural differences by cultivating the cultural empathy ability of communicators and enhancing their cultural awareness and some other ways.

\section{Conclusions}

As all stated that, in the past 20 years, the study of cross-cultural communication in China is particularly concerned with the definition of cross-cultural communication competence, foreign language teaching, cultural differences and communicative strategies. Among them, foreign language teaching has always been a hot research topic; In addition, the empirical research on cross-cultural sensitivity or cross-cultural communicative competence and how to cultivate intercultural communicative competence have become a hot research topic in recent years. The most prominent author and core literature in cross-cultural communication research is $\mathrm{Hu}$ Wenzhong and his related literature.

Cross-cultural communication research in China has still not formed a scale and there are still many problems. For instance, the research content is narrow and there are too many theoretical studies and only a few empirical studies. The author hoped that this study can help scholars timely acknowledge hot issues and frontiers of cross-cultural communication research to improve the quality of researches in this field.

\section{Acknowledgement}

This research was financially supported by the Humanities and Social Sciences Project of Hubei Provincial Department of Education, ID $13 \mathrm{y} 021$.

\section{References}

[1] M. J. Bennett, A developmental approach to training for intercultural sensitivity, International Journal of Intercultural relations. 1986.10(2):179-196. 
[2] Jiwan $\mathrm{Bi}$, The main task of the second language is to cultivate students' cross-cultural communicative competence, Foreign Language in China. 2005(1): 66-70.

[3] Guoming Chen, The generation and the development of "cross-cultural communication" as a term and a discipline, Academic research. 2010: 141-148

[4] Yun Cai, Exploration of training strategy on college students' oral English communicative competence, English Square. 2016:95-96

[5] Wenzhong $\mathrm{Hu}$, Crossing cultural barriers, Foreign Language Teaching and Research Press. 2002

[6] Xiaoli Jiang, Study on the difficulties and strategies of Chinese oral communication for American College Students, Chinese Teaching in the World. 2015:250-265

[7] Jingjing Kong, A study on the teaching of communicative strategies, Foreign Language World. 2004 (5)

[8] Jingjing Kong, An investigation of communication strategies used by Chinese non-English major postgraduates, English Language Teaching in China. 2006 (2)

[9] Yan Li et Weidong Zhang, The construction of cross-cultural communicative competence training mode for foreign language majors-based on CDIO teaching concept, Computer-assisted Foreign Language Education. 2013:61-65 